\title{
Pre dialysis of blood prime in continuous hemodialysis normalizes pH and electrolytes
}

Received: 20 March 2003 / Revised: 13 June 2003 / Accepted: 13 June 2003 / Published online: 2 October 2003 (C) IPNA 2003

\begin{abstract}
In critically ill children weighing $<10 \mathrm{~kg}$, it is necessary to use blood as a priming solution for the extracorporeal continuous renal replacement therapy (CRRT) circuit before initiating CRRT to prevent hemodilution and maintain adequate oxygenation. However, blood bank blood usually contains supra-physiological electrolyte concentrations and a non-physiological acidbase balance that may exacerbate the patient's condition. The objective of this trial was to develop a simple protocol to pre-treat blood bank-derived blood to yield a more physiological blood priming solution. Expired human blood in a recirculating in vitro CRRT circuit was dialyzed prior to the initiation of CRRT using a physiological dialysate solution. Serial blood samples were assessed for electrolyte and $\mathrm{pH}$ content. Regimens using maximal blood flow rates $(180-200 \mathrm{ml} / \mathrm{min})$ and aggressive dialysate flow rates $(33-42 \mathrm{ml} / \mathrm{min})$ were able to correct severely hyperkalemic and acidemic blood within $7.5 \mathrm{~min}$. Initially elevated blood potassium concentrations $>20 \mathrm{mEq} / \mathrm{l}$ were normalized to below $5 \mathrm{mEq} / \mathrm{l}$ within $7.5 \mathrm{~min}$ of dialysis in all cases. Blood bank-derived blood can be "conditioned" quickly to physiological $\mathrm{pH}$ and electrolyte concentrations using these simple pre-dialysis regimens. Unlike some blood
\end{abstract}

This manuscript was presented in poster form on 3 March 2003 at the 8th International Conference on Continuous Renal Replacement Therapies, San Diego, California, USA

D. A. Pasko · B. A. Mueller

College of Pharmacy, University of Michigan,

Michigan, USA

T. A. Mottes

Pediatric Dialysis Unit, University of Michigan Health Systems, Ann Arbor, Michigan, USA

D. A. Pasko (๘)

Department of Pharmacy Services,

B2D301 University Hospital,

1500 E. Medical Center Drive, Ann Arbor, MI 48109-0008, USA

e-mail: dapasko@umich.edu

Tel.: +1-734-9368210

Fax: +1-734-9367027 preparation regimens that have been published, the technique used in this trial requires no special equipment or added medications that are not already used in CRRT.

Keywords Blood prime - Continuous renal replacement therapy $\cdot$ Extracorporeal membrane oxygenation

\section{Introduction}

Newer extracorporeal therapies are being used increasingly in pediatric and neonatal intensive care units to treat critically ill children. Therapies such as continuous renal replacement therapy (CRRT) are becoming commonplace. A difficulty with CRRT circuits used in pediatric patients is the substantial volume $(\sim 100 \mathrm{ml})$ required to fill the blood side of the extracorporeal circuit. In small children weighing $<10 \mathrm{~kg}$, this necessitates using blood from the blood bank as a prime in the extracorporeal circuit because the volume in the circuit represents a substantial fraction of the patient's circulating blood volume. Priming the circuit with saline or albumin does not adequately replace the lost blood volume, which can account for $\sim 10 \%$ of the child's total blood volume. In larger patients, priming solutions such as normal saline, lactated Ringer's, or albumin solution can be used successfully, but in these small patients, only the use of blood prevents significant hemodilution when CRRT is initiated.

The use of blood as the extracorporeal priming solution may carry deleterious consequences. Blood bank-derived blood may be acidemic, hyperkalemic, and hypocalcemic. Any of these factors can lead to significant hemodynamic instability when this blood prime is infused into the patient at the initiation of CRRT. In addition, these factors become increasingly important when using the AN-69 hemofilter (Hospal, Lyon, France). When acidemic blood comes into contact with the AN-69 membrane, patients manifest symptoms consistent with bradykinin release [1]. Regardless of hemofilter membrane type, another factor that may cause hemodynamic 
instability is the hypocalcemia caused by the citrate anticoagulant added to blood bank blood. The anticoagulant effect of citrate is due to its ability to bind free calcium, resulting in blood that cannot form clots. Consequently, infusion of these citrated blood primes without adequate calcium supplementation, via infusion or added to the dialysate, could result in severe hypocalcemia in small children receiving CRRT.

Strategies to pre-treat this blood prime to reduce adverse reactions in neonatal cardiopulmonary bypass (CPB) have been proposed [2, 3]. Journois et al. [3] hemofiltered the blood prime prior to initiating CPB and then replaced the removed volume with a physiological electrolyte solution. Nagashima et al. [2] also hemofiltered blood prior to CPB and replaced the removed volume with a maltose-, glucose-, and electrolyte-containing solution. In both cases, the authors reported some benefit to the technique.

Blood prime pre-treatment has not been reported for pediatric CRRT, although alternative strategies have been used to avoid the deleterious consequences with blood prime infusion [1] (Table 1). In the absence of a simple procedure, there should be careful monitoring for blood prime infusion-related effects (especially when an AN-69 hemofilter is used). These must be treated aggressively through the use of calcium and sodium bicarbonate administration. The administration of these two agents results in the formation of a precipitate, so these drugs should be infused at different vascular access points. Given that vascular access sites are not easily placed in these small infants, particularly when CRRT is being started, blood prime conditioning regimens that would obviate these additional infusions and prevent adverse reactions to the blood prime would be valuable.

We report the results of an in vitro blood prime conditioning regimen that dialyzes the blood prime prior to initiation of therapy. This regimen was performed with two of the most commonly used CRRT machines and may provide benefit to critically ill neonates and small children requiring CRRT. This technique does not use hemofiltration and requires no special equipment or added medications not already used in CRRT. The purpose of this study was to determine the efficacy of a CRRT bloodconditioning regimen for use prior to initiation of CRRT in children.

\section{Materials and methods}

An in vitro CRRT circuit set in the continuous venovenous hemodialysis mode using blood bank-derived blood was used. Blood was continually re-circulated while the dialysate flowed in a non-recirculation (single-pass) mode in a countercurrent direction. Blood priming regimens were developed for two of the most commonly used CRRT machines on the market, the Diapact CRRT machine (B. Braun Medical, Bethlehem, Pa., USA) and the Prisma CRRT machine (Gambro USA, Lakewood, Colo., USA).

\section{Dialysate}

The dialysate used in all experiments was the same solution as is used for pediatric continuous venovenous hemodialysis (CVVHD) at our institution. This custom-made dialysate solution contained sodium chloride $105 \mathrm{mEq} / \mathrm{l}$, sodium bicarbonate $35 \mathrm{mEq} / \mathrm{l}$, sodium phosphate $1.5 \mathrm{mmol} / \mathrm{l}$, magnesium sulfate $0.75 \mathrm{mEq} / \mathrm{l}$, and potassium chloride $3 \mathrm{mEq} / \mathrm{l}$. Dialysate was used during priming on the dialysate side for both experiments.

\section{Diapact priming experiments \\ Diapact blood priming regimen}

The Diapact CRRT machine (B.Braun Medical) was programmed for CVVHD mode. The hemodiafilter used for each of the four experiments was a new, non-reused Multiflow-60 hemodiafilter (Hospal, Lyon, France) and the tubing was a standard Diapact CRRT tubing kit (B.Braun Medical). The blood side volume of the tubing and hemofilter was $105 \mathrm{ml}$. The arterial side of the extracorporeal circuit was primed with $0.9 \%$ sodium chloride containing heparin 5 units/ml according to the manufacturer's protocol. Using heparinized saline on the blood side and the custom dialysate on the dialysate side, the Diapact ran its internal priming regimen.

\section{Diapact blood preparation}

Expired unfiltered human packed red blood cells (PRBCs) were obtained from the hospital blood bank and were diluted with $0.9 \%$ normal saline to a hematocrit of approximately $40 \%$. The diluted

Table 1 Blood prime conditioning approaches (CRRT continuous renal replacement therapy)

\begin{tabular}{|c|c|c|c|c|}
\hline & Resuscitation & Blood prime bypass $[3]$ & Extracorporeal techniques & \\
\hline Technique & $\begin{array}{l}\text { THAM and calcium } \\
\text { added to blood } \\
\text { prime [7] }\end{array}$ & $\begin{array}{l}\text { Saline used as prime and } \\
\text { discarded, replacement } \\
\text { blood infused post filter }\end{array}$ & Hemofiltration of blood prime $[1,2,6]$ & $\begin{array}{l}\text { Hemodialysis } \\
\text { of blood prime }\end{array}$ \\
\hline $\begin{array}{l}\text { Published use } \\
\text { for CRRT? }\end{array}$ & Yes & Yes & No & This report \\
\hline Costs & $\begin{array}{l}\text { Some drugs, } \\
\text { mostly personnel }\end{array}$ & $\begin{array}{l}\text { Tubing, stopcocks, drugs, } \\
\text { mostly personnel }\end{array}$ & $\begin{array}{l}\text { Custom-made ultrafiltrate replacement } \\
\text { solution, requires in-line hemofiltration } \\
\text { system, mostly personnel }\end{array}$ & $\begin{array}{l}\text { No extra equipment } \\
\text { or drugs needed, } \\
\text { mostly personnel }\end{array}$ \\
\hline $\begin{array}{l}\text { Electrolytes } \\
\text { normalized? }\end{array}$ & No & No & $\begin{array}{l}\text { Dependent on ultrafiltrate replacement } \\
\text { solution content }\end{array}$ & Yes \\
\hline pH normalized? & Unknown & No & $\begin{array}{l}\text { Dependent on ultrafiltrate replacement } \\
\text { solution } \mathrm{pH}\end{array}$ & Yes \\
\hline
\end{tabular}


blood was transferred to an empty plastic intravenous bag (Abbott, Chicago, Ill., USA) and warmed in a water bath to $37^{\circ} \mathrm{C}$.

When the machine's priming regimen was completed, blood replaced the saline in the circuit using the following technique. The bag of blood was spiked with a dispensing spike (B.Braun), and a three-way stopcock (Medex, Hilliard, Ohio, USA) was attached to the spike (B. Braun Medical) via a Luer-lock connection. The arterial arm of the extracorporeal circuit was attached to one of the stopcock ports. The stopcock was opened so that blood could flow into the arterial line. The blood pump was started and as blood was pumped through the circuit the blood-side saline collected in the venous waste bag. This was continued until blood reached the end of the venous limb of the circuit. At this point the pumps were stopped; the venous line was clamped and was detached from the waste bag and connected to the remaining stopcock port. The stopcock was then turned so that the remaining blood in the bag was blocked and the venous blood flowed through the stopcock back into the arterial arm of the circuit. At this point, the venous line was unclamped and the blood pumps were restarted in a recirculating manner without any dialysate flow (bypass mode). Blood was re-circulated for approximately $5 \mathrm{~min}$ and then the machine was placed into CVVHD therapy mode. Once all pumps were running at the desired speed, the experiment was initiated.

The dialysis parameters for the experiment were blood $\left(\mathrm{Q}_{\mathrm{b}}\right)$ and dialysate $\left(\mathrm{Q}_{\mathrm{d}}\right)$ flows of $200 \mathrm{ml} / \mathrm{min}$ and $33.3 \mathrm{ml} / \mathrm{min}(2 \mathrm{l} / \mathrm{h})$, respectively. No ultrafiltrate was formed. These are not the flow rates that typically would be used in a small child, but were chosen because they could be achieved with this machine and would minimize the time necessary to condition the blood before CVVHD initiation in a small child. The $\mathrm{Q}_{b}$ and $\mathrm{Q}_{\mathrm{d}}$ capacity of the Diapact can actually go much faster than this but are actually higher than what is usually used clinically in our institution. We chose the experimental flow rates similar to those we use in adult patients in our institution.

Prisma priming experiments

Prisma blood priming regimen

The Prisma (Gambro USA) was programmed for CVVHD mode. Two experiments were conducted with the Prisma system. The hemodiafilter used for each of the two experiments was a new, nonreused Multiflow-60 hemodiafilter (Hospal) and the tubing was a standard Prisma CRRT tubing kit (Gambro USA). The blood side volume of the tubing and hemofilter is $98 \mathrm{ml}$. The arterial side of the extracorporeal circuit was primed with $0.9 \%$ sodium chloride containing heparin 5 units $/ \mathrm{ml}$ according to the manufacturer's protocol. Using heparinized saline on the blood side and the custom dialysate on the dialysate side, the Prisma ran its internal priming regimen.

\section{Prisma blood preparation}

Expired unfiltered human whole blood (hematocrit 40\%) from the University of Michigan Blood Bank was used in both Prisma trials. Once the internal Prisma priming procedures were completed, the whole blood was pumped into the arterial arm of the Prisma extracorporeal circuit, as described for the Diapact circuit methods.

The dialysis parameters for the experiment were blood $\left(\mathrm{Q}_{\mathrm{b}}\right)$ and dialysate $\left(\mathrm{Q}_{\mathrm{d}}\right)$ flows of $180 \mathrm{ml} / \mathrm{min}$ and $41.67 \mathrm{ml} / \mathrm{min}(2.5 \mathrm{l} / \mathrm{h})$, respectively. No ultrafiltrate was formed. These are not the flow rates that would be used in a small child, but were chosen because they were the maximum flow rates that could be achieved with this machine and would minimize the time necessary to condition the blood before CVVHD initiation in a small child.
Sampling strategy

The same sampling regimen was used in both the Prisma and Diapact experiments. A $1.5-\mathrm{ml}$ sample of blood was taken immediately before any experiment was started. Once the hemodialysis had begun, $1.5-\mathrm{ml}$ blood samples were taken from the arterial, venous, and effluent ports, at 2.5, 5, 7.5, 10, 15, 20, and $30 \mathrm{~min}$. Samples were transferred to SST Vacutainers (BectonDickson, Franklin Lakes, N.J., USA), and centrifuged at 2,000 rpm for $30 \mathrm{~min}$. The plasma was transferred to Cryovials (Fisher Scientific, Pittsburgh, Pa., USA) and frozen at $-80{ }^{\circ} \mathrm{C}$ until analyzed.

Electrolyte and $\mathrm{pH}$ analysis

Serum samples were analyzed via an indirect ion-selective electrode (ISE) using an Integra 400 Plus chemical analyzer (Roche, Indianapolis, Ind., USA). The coefficient of variance was $<1.5 \%$ for all electrolytes analyzed. All samples were run in duplicate. The $\mathrm{pH}$ of the blood $\mathrm{pH}$ meter, Corning 430, Fisher Scientific) was assessed immediately before starting each experiment (time zero) and again after $30 \mathrm{~min}$ of dialysis.

\section{Calculations}

Electrolyte clearance was calculated as: $\mathrm{K}=(\mathrm{E} / \mathrm{P}) \times \mathrm{Qe}$ where $\mathrm{K}=$ solute clearance, $\mathrm{E}=$ solute concentration in the effluent, $\mathrm{P}=$ solute concentration of the blood, and $\mathrm{Qe}=$ flow rate of the effluent (spent dialysate).

Electrolyte clearance $(\mathrm{K})$ was assessed at each sampling time. Results of the clearance data are expressed as the mean \pm SD value of each of these calculated values.

\section{Pharmacokinetic calculation}

Determination of the time it took for the concentration of the solute in the blood to decline halfway from the recirculating blood prime concentration to the concentration of the fresh dialysate flowing in a single pass mode was calculated as: time for blood concentration to get to $1 / 2$ the difference between the blood prime and the dialysate $=0.693$ (volume of the extracorporeal circuit blood volume)/dialysate flow rate.

The application of this equation in humans is inappropriate because a drug's clearance and distribution volume are independent and a perturbation of one often causes a change in half life without changing the other parameter [4]. However, for the purposes of this closed system with a fixed volume and clearance that is set by a fixed pump rate, this equation works well and is appropriate.

\section{Results}

Initial blood electrolyte concentrations and $\mathrm{pH}$ were not physiological. For example, initial blood potassium in these samples, prepared from expired PRBC ranged from 9.6 to $21.3 \mathrm{mEq} / \mathrm{l}$ in the Diapact experiments and from 24.1 to $25.8 \mathrm{mEq} / 1$ in the Prisma experiments. Initial sodium and chloride values were also higher than normal blood values. Mean blood pH at baseline was 6.50 in the Diapact and 6.35 in the Prisma experiments. At the end of the 30-min experiment the blood $\mathrm{pH}$ was 7.40 for all experiments. Serial electrolyte values were obtained and appear in Figs. 1 and 2.

At the $\mathrm{Q}_{\mathrm{b}}$ and $\mathrm{Q}_{\mathrm{d}}$ used in these experiments, electrolytes were within normal physiological concentrations 
Fig. 1 a Potassium concentrations for the Diapact experiments $1-4$ over time (top graph). b Sodium and chloride concentrations for the Diapact experiments $1-4$ over time (bottom graph)
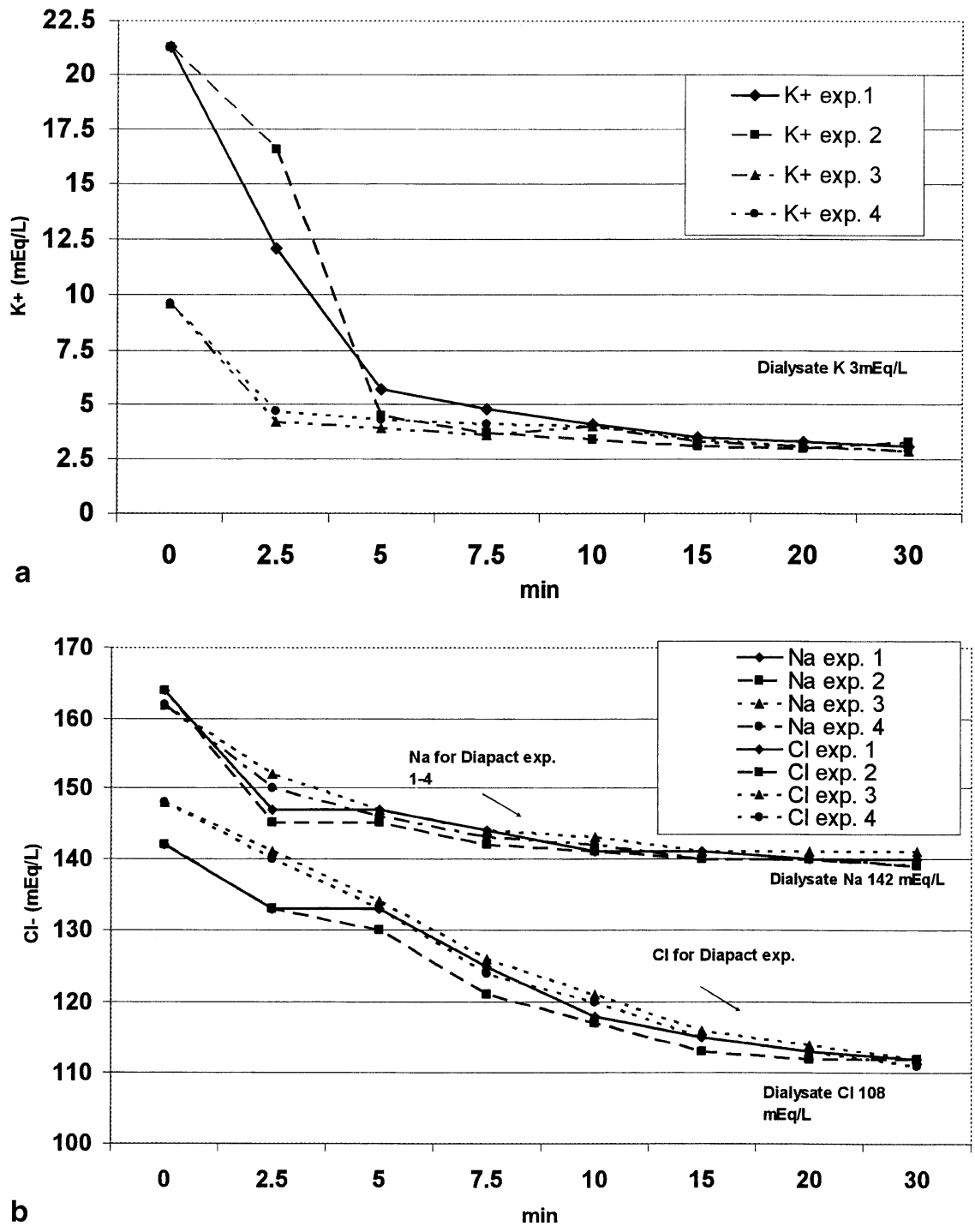

within 5-7.5 min. This was true even in situations where the initial potassium concentrations were as much as fivefold above "normal" concentrations. Electrolyte clearance approximated $\mathrm{Q}_{\mathrm{d}}$ in these experiments. In the Diapact experiments with a $\mathrm{Q}_{\mathrm{d}}$ of $33.3 \mathrm{ml} / \mathrm{min}$, the mean \pm SD potassium clearance, sodium clearances, and chloride clearances were $30.9 \pm 2.8, \quad 33.4 \pm 0.1$, and $33.3 \pm 0.2 \mathrm{ml} / \mathrm{min}$, respectively. The mean $\pm \mathrm{SD}$ clearances in the Prisma experiments with a $\mathrm{Q}_{\mathrm{d}}$ of $41.7 \mathrm{ml} / \mathrm{min}$ for potassium, sodium, and chloride were $36.41 \pm 4.0,39.1 \pm$ 4.3 , and $42.8 \pm 5.2 \mathrm{ml} / \mathrm{min}$, respectively.

\section{Discussion}

The electrolyte clearance approximated $\mathrm{Q}_{\mathrm{d}}$ in all experiments. The $\mathrm{Q}_{\mathrm{b}}$ and $\mathrm{Q}_{\mathrm{d}}$ used in the Prisma experiment were the maximum achievable with this system. The flow rates used in the Diapact arm of the trial were similar to rates used in treating adults, but much higher $\mathrm{Q}_{\mathrm{b}}(500 \mathrm{ml} / \mathrm{min})$ and $\mathrm{Q}_{\mathrm{d}}(200 \mathrm{ml} / \mathrm{min})$ can be achieved with this machine. Consequently, it may be that blood conditioning can be achieved with the Diapact even faster than we accomplished.

Prior to the study, we mathematically modeled the flow rates in these experiments to provide an estimate of the time required to modify the electrolyte content of the blood to that of the dialysate. We developed a nontraditional use of "half-life" to visualize the data. Typically, the half-life of a compound is the time that is required to reduce the compound's concentration halfway to zero. In this experiment, the compounds of interest would not continually approach zero like typical firstorder drug elimination or radioactive decay. Instead, the concentration of the compound in the blood would continually approach the concentration of the compound in the dialysate. This assumption is true as long as the supply of dialysate is unlimited, the blood volume is fixed, and the compound is not being generated within the blood. In the present experiment, which used a closed 
Fig. 2 a Potassium concentrations for the Prisma experiments 1 and 2 over time (top graph). b Sodium and chloride concentrations for the Prisma experiments 1 and 2 over time (bottom graph)

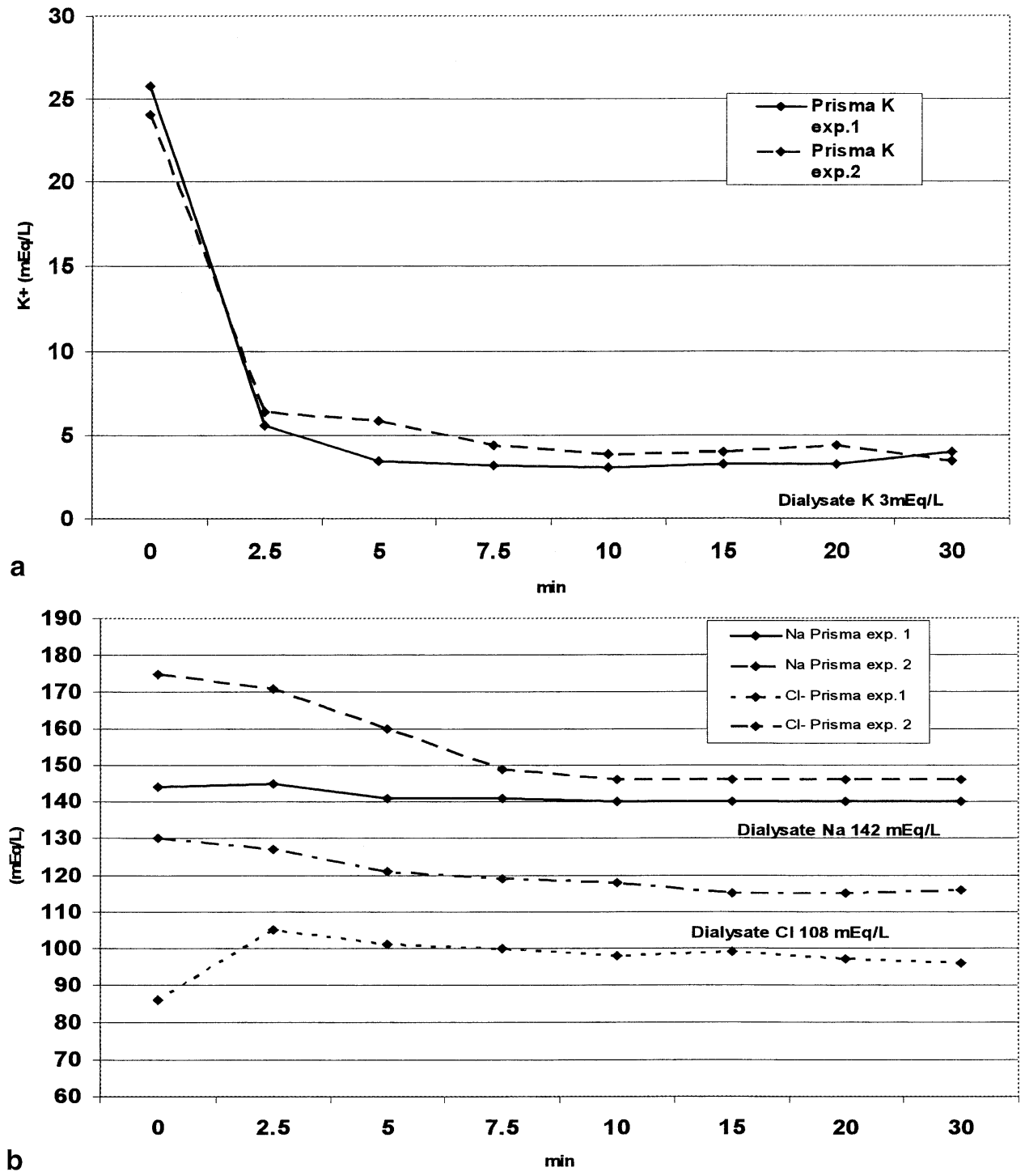

system with a fixed volume and clearance that is set by a fixed pump rate, this equation works well. Indeed, inserting the known blood side volume of the prime (98 $\mathrm{ml}$ Prisma or $105 \mathrm{ml}$ Diapact) and the $\mathrm{Q}_{\mathrm{d}}$ used in each experiment $(41.7 \mathrm{ml} / \mathrm{min}$ Prisma or $33.3 \mathrm{ml} / \mathrm{min}$ Diapact) results in a predicted time for the blood concentration to get to half the difference between the blood prime and the dialysate of 1.62 or $2.19 \mathrm{~min}$, respectively. Experimentally, this time was nearly identical to the calculated values. Each of these "half-lives" brings the blood solute content $50 \%$ closer to the fresh dialysate concentrations. Consequently, after 4 or 5 half-lives $(8-10 \mathrm{~min})$ the blood electrolyte content should be nearly identical to that of fresh dialysate.

This experiment used blood with a very high initial potassium concentration, possibly because they were from expired whole blood (Prisma experiments) or PRBC (Diapact experiments). Nonetheless, Brophy et al. [1] reported that there are high concentrations of potassium in the blood prime when non-expired blood bank PRBCs are used. CRRT initiation rapidly delivers a substantial potassium bolus to these critically ill children. For example, a non-pre-dialyzed $100-\mathrm{ml}$ blood prime with a potassium concentration of $20 \mathrm{mEq} / \mathrm{l}$ could be infused at a rate of $10 \mathrm{ml} / \mathrm{min}$ into a $2-\mathrm{kg}$ critically ill child, resulting in $2 \mathrm{mEq}$ of potassium infused within $10 \mathrm{~min}$. This may lead to cardiac instability. Pre-dialysis of the blood prime would eliminate this potassium bolus. In countries outside of the United States, clinicians may have the option of using a smaller, more infant-oriented tubing system with a smaller blood side volume. For example, the Prisma M10 Pre Set system (Gambro, Lyon, France) contains a 0.042$\mathrm{m}^{2}$ AN69 hemodiafilter and a PVC tubing kit with a total blood side volume of $50 \mathrm{ml}$. This kit would require a smaller blood prime but at the cost of having to use a hemofilter with a surface area that is substantially smaller than we used in our study $\left(0.6 \mathrm{~m}^{2}\right)$.

The anticoagulant used in whole blood and PRBC is citrate, which binds to free calcium and prevents clotting. Consequently, the infusion of these blood primes delivers 
unbound citrate and almost no free calcium. The unbound citrate will bind calcium within the patient's blood once infused. Typically, either a separate calcium infusion or calcium-containing dialysate is necessary to counter this hypocalcemic effect in very small children. In settings utilizing citrate anticoagulation, a calcium-free dialysate is commonly used. A possible salutary effect of the blood conditioning technique presented here is that citrate is likely removed from the blood via dialysis because free citrate and calcium citrate have a very small molecular weight. Pediatric studies have documented that citrate is easily removed by CRRT [5]. However, the calcium content of pre-dialyzed blood as used in this experiment would not provide calcium either because the citratebound calcium is also removed by the pre-dialysis technique. Therefore, it is imperative that a calcium infusion be started at the initiation of CRRT to counteract the hypocalcemic blood prime using the described technique, unless a calcium-containing dialysate is used in the conditioning regimen.

Bradykinin formation resulting from blood prime contact with an AN69 hemofilter membrane is likely to be the cause of the most serious adverse reaction associated with CRRT in pediatric patients. Brophy et al. [1] hypothesized that hemodynamic instability may result from the infusion of a PRBC-based blood prime because of the low $\mathrm{pH}$ of this blood. These authors suggested that it is the addition of an acidemic blood prime that causes an acute bradykinin release and that this is responsible for the AN69 membrane hypersensitivity reaction. In that report the blood prime $\mathrm{pH}$ was 6.1-6.4 and the $0.9 \%$ saline PRBC diluent had a $\mathrm{pH}$ of 5.9. In our study, we reconstituted the PRBCs as did Brophy et al. [1] and the $\mathrm{pH}$ after reconstitution was 6.5. The $\mathrm{pH}$ of the expired whole blood in the present study was 6.35. Our dialysate had a pH of 8.05 , and after $30 \mathrm{~min}$ of the bloodconditioning regimen the blood prime $\mathrm{pH}$ was 7.4 in both the Diapact and Prisma studies. Although we used an AN69 hemofilter in our studies, we did not directly measure bradykinin blood concentrations. If an acidemic blood prime is the cause for the AN69 membrane hypersensitivity reaction, this blood-conditioning regimen may eliminate this problem associated with CRRT initiation. Furthermore, it is possible that this bloodconditioning regimen could remove bradykinin directly. The molecular weight of bradykinin (1,060 daltons) is such that it may be removed by CVVHD. These studies remain to be performed.

Other blood-conditioning techniques have been suggested for extracorporeal therapies. Nagashima et al. [2] reported a blood-conditioning regimen to be used prior to CBP procedures. In this regimen, $800 \mathrm{ml}$ whole blood was circulated in the CBP circuit and then $3,300 \mathrm{ml}$ of a "hemofiltration" solution was added to the circuit. This hemofiltration solution was then removed by hemofiltration at $250 \mathrm{ml} / \mathrm{min}$ using a highly permeable hemofilter (HF 1.0U, Toray, Japan). This technique was documented to reduce circuit concentrations of potassium, sodium, and bradykinin. Once CBP was instituted, patients tended to have better initial hemodynamics than a group that did not have conditioned blood prior to initiation of CBP. Blood $\mathrm{pH}$ values were not reported in this study. The hemofiltration solution in this study was not a physiological solution and the treated blood became very hypocalcemic and thrombocytopenic.

Others have proposed that ultrafiltration during or immediately after cardiac bypass have had an effect on mediators such as tumor necrosis factor (TNF), interleukin-1 (IL-1), interleukin-8 (IL-8), and complement, C3 a $[2,3,6,7]$. Journois et al. [3] found that using highvolume, zero-balanced hemofiltration during the rewarming period of CBP in children could reduce $\mathrm{C} 3$ a and TNF. In this study, two groups of patients were randomized to either receive no hemofiltration or zerobalanced hemofiltration using a polyacrylonitrile hemofilter (SA $0.6 \mathrm{~m}^{2}$, Multiflow, Hospal Laboratories, Meyzieu, France). The volume of ultrafiltration during the re-warming period in the hemofiltration group was $1,960 \mathrm{ml} / \mathrm{m}^{2}$. The patients in the hemofiltration arm also showed significant reduction of IL-1, IL-6, IL-8, and myelo-oxidase at $24 \mathrm{~h}$. Similar results with regards to TNF removal have been reported by Millar et al. [6]. This study showed a significant decrease in TNF plasma concentrations before and after hemofiltration, and considerable amounts of TNF were detected in the ultrafiltrate [6]. Convective therapies should be better at removing inflammatory mediators than purely diffusive therapies as in our pre-dialysis of the blood prime technique; however, quantification of mediator clearance in our study was not assessed.

$\mathrm{CPB}$ is known to be associated with numerous adverse events that can manifest as a generalized inflammatory response. This response involves the activation of several inflammatory mediators. Hemofiltration seems to play a role in reducing inflammatory mediators, but whether this is due to convective removal or binding to the membrane, or both, is unknown. Some studies have shown the actual removal of mediators, and have detected TNF in the ultrafiltrate, while other studies suggest that the recirculation of the primed blood during ultrafiltration may actually enhance the adherence of the larger inflammatory mediators to the hemofilter. Regardless of mechanism, patients that receive hemofiltration during or after $\mathrm{CPB}$ have been shown to have lower levels of inflammatory mediators, which ultimately may enhance patient recovery.

Patients receiving extracorporeal membrane oxygenation (ECMO) also receive a large blood prime and can face similar electrolyte and acidosis issues. The University of Michigan ECMO team uses a blood-conditioning regimen consisting of a buffering cocktail made with TRIS-hydroxy-methyl aminomethane (THAM), calcium chloride, heparin, with equal parts of PRBC and a solution of sterile water and sodium bicarbonate. This solution helps normalize the $\mathrm{pH}$ and calcium. The hematocrit is slightly lowered. This system helps the $\mathrm{pH}$; however, hyperkalemia can still be a problem. Another approach is using a "Bypass System" described by Brophy et al. [1] 
This technique uses stopcocks in series that allows the saline prime to be discarded, and PRBCs, diluted 1:1 with saline, are directly infused into the venous return catheter port. This method minimizes the initial blood prime exposure to the hemodiafilter, which may reduce overall bradykinin formation. However, this method does not change the potassium content or the $\mathrm{pH}$ of the PRBCs that are directly given to the patient. If the weight of the child is $<3 \mathrm{~kg}$, the PRBC mixture is markedly hyperkalemic and acidemic, making it difficult for the child's small blood volume to help normalize the potassium and $\mathrm{pH}$. Furthermore, this method warrants prudence because of the use of the citrated PRBC. A supplemental calcium infusion should be started before connecting the patient to the pre-dialyzed blood prime to prevent hypocalcemia from the blood prime that has been cleared of calcium.

Although not done in our in vitro experiment, in the clinical setting it would also be essential to filter the blood as specified in the institution's guidelines. In critically ill patients receiving CRRT, there is a possibility of renal function not recovering to a life-sustaining capacity. Consequently, kidney transplantation might be necessary. Filtered blood may minimize the kidney recipient's risk of inducing sensitization to the HLA antigens of the donor.

In conclusion, based on the results of this experiment, it is concluded that using this pre-dialysis method of blood priming, blood electrolyte concentrations can be normalized within approximately 7.5-10 min when using a physiologically compatible dialysate solution. This system required very little time and no extra equipment, tubing, monitoring, or drugs to complete. This technique worked equally well for diluted PRBCs and whole blood.
Further studies that measure inflammatory mediator concentrations using this technique are necessary before it is attempted in children.

Acknowledgements The authors acknowledge the contributions of Dr. Robertson D. Davenport for providing the blood products for this trial and of B. Braun Medical Incorporated for providing materiel support.

\section{References}

1. Brophy PD, Mottes TA, Kudelka TL, McBryde KD, Gardner JJ, Maxvold NJ, Bunchman TE (2001) AN-69 membrane reactions are $\mathrm{pH}$-dependent and preventable. Am J Kidney Dis 38:173-178

2. Nagashima M, Imai Y, Seo K, Terada M, Aoki M, Shinoka T, Koide M (2000) Effect of hemofiltrated whole blood pump priming on hemodynamics and respiratory function after the arterial switch operation in neonates. Ann Thorac Surg 70:1901-1906

3. Journois D, Israel-Biet D, Pouard P, Rolland B, Silvester W, Vouhe P, Safran D (1996) High-volume, zero-balanced hemofiltration to reduce delayed inflammatory response to cardiopulmonary bypass in children. Anesthesiology 85:965-976

4. Evans WE, Schentag JJ, Jusko WJ (1992) Applied pharmacokinetics, principles of therapeutic drug monitoring, 3rd edn. Applied Therapeutics, Vancouver, Wash, pp 2-29

5. Chadha V, Garg U, Warady BA, Alon US (2002) Citrate clearance in children receiving continuous venovenous renal replacement therapy. Pediatr Nephrol 17:819-824

6. Millar AB, Armstrong L, Linden J van der, Moat N, Ekroth R, Westwick J, Scallan M, Lincoln C (1993) Cytokine production and hemofiltration in children undergoing cardiopulmonary bypass. Ann Thorac Surg 56:1499-1502

7. Plotz FB, Oeveren W van, Bartlett RH, Wildevuur CR (1993) Blood activation during neonatal extracorporeal life support. Thorac Cardiovasc Surg 105:823-832 